\title{
PERIODIC SOLUTIONS OF A TWO-SPECIES RATIO-DEPENDENT PREDATOR-PREY SYSTEM WITH TIME DELAY IN A TWO-PATCH ENVIRONMENT
}

\author{
ZHENGQIU ZHANG ${ }^{1}$ and ZHICHENG WANG ${ }^{1}$
}

(Received 14 September, 2001; revised 7 January, 2002)

\begin{abstract}
By using the continuation theorem of coincidence degree theory, a sufficient condition is obtained for the existence of a positive periodic solution of a predator-prey diffusion system.
\end{abstract}

\section{Introduction}

$\mathrm{Xu}$ and Chen [4] considered a two-species ratio-dependent predator-prey diffusion model with time delay given by

$$
\begin{aligned}
& x_{1}^{\prime}(t)=x_{1}(t)\left(a_{1}-a_{11} x_{1}(t)-\frac{a_{13} x_{3}(t)}{m x_{3}(t)+x_{1}(t)}\right)+D_{1}\left(x_{2}(t)-x_{1}(t)\right) \\
& x_{2}^{\prime}(t)=x_{2}(t)\left(a_{2}-a_{22} x_{2}(t)\right)+D_{2}\left(x_{1}(t)-x_{2}(t)\right) \\
& x_{3}^{\prime}(t)=x_{3}(t)\left(-a_{3}+\frac{a_{31} x_{1}(t-\tau)}{m x_{3}(t-\tau)+x_{1}(t-\tau)}\right)
\end{aligned}
$$

where $x_{i}(t)$ represents the prey population in the $i^{i \text { th }}$ patch, $i=1,2$, and $x_{3}(t)$ represents the predator population. Here $\tau>0$ is a constant delay due to gestation, $D_{i}$ is a positive constant denoting the dispersal rate, $i=1,2$, and $a_{i}(i=1,2,3), a_{11}, a_{13}, a_{22}, a_{31}$ and $m$ are positive constants.

In $\mathrm{Xu}$ and Chen [4], the local and global asymptotical stability of the positive equilibrium of the system (1.1) were studied. For an ecological interpretation of system (1.1), we refer to [4] and references cited therein.

\footnotetext{
'Department of Applied Mathematics, Hunan University, Changsha, Hunan 410082, P. R. China; e-mail: zcwang@hnu.net.cn.

(C) Australian Mathematical Society 2003, Serial-fee code 1446-1811/03
} 
Realistic models require the inclusion of the effect of change in the environment. This motivates us to consider the following two species predator-prey diffusion model with time delay:

$$
\left.\begin{array}{rl}
x_{1}^{\prime}(t)= & x_{1}(t)\left(a_{1}(t)-a_{11}(t) x_{1}(t)-\frac{a_{13}(t) x_{3}(t)}{m(t) x_{3}(t)+x_{1}(t)}\right) \\
& +D_{1}(t)\left(x_{2}(t)-x_{1}(t)\right), \\
x_{2}^{\prime}(t)= & x_{2}(t)\left(a_{2}(t)-a_{22}(t) x_{2}(t)\right)+D_{2}(t)\left(x_{1}(t)-x_{2}(t)\right), \\
x_{3}^{\prime}(t)= & x_{3}(t)\left(-a_{3}(t)+\frac{a_{31}(t) x_{1}(t-\tau)}{m(t) x_{3}(t-\tau)+x_{1}(t-\tau)}\right) .
\end{array}\right\}
$$

In addition, the effects of a periodically changing environment are important for evolutionary theory as the selective forces on systems in a fluctuating environment differ from those in a stable environment. Therefore the assumptions of periodicity of the parameters are a way of incorporating the periodicity of the environment (for example, seasonal changes, food supplies, mating habits, and so on), which leads us to assume that $D_{i}(i=1,2), a_{i}(i=1,2,3), a_{11}, a_{13}, a_{22}, a_{31}$ and $m$ are strictly positive continuous $w$-periodic functions.

As pointed out by Freedman and Wu [1] and Kuang [3], it is of interest to study the global existence of periodic solutions for systems representing predator-prey or competition systems. In this paper, our aim is to use the continuation theorem of coincidence degree theory which was proposed in [2] by Gaines and Mawhin to establish the existence of at least one positive $w$-periodic solution with $w>0$ of system (1.2).

Let $X, Z$ be real Banach spaces, $L: \operatorname{dom} L \subset X \rightarrow Z$ a Fredholm mapping of index zero and $P: X \rightarrow X, Q: Z \rightarrow Z$ continuous projectors such that $\operatorname{Im} P=\operatorname{Ker} L, \operatorname{Ker} Q=\operatorname{Im} L, X=\operatorname{Ker} L \oplus \operatorname{Ker} P$ and $Z=\operatorname{Im} L \oplus \operatorname{Im} Q$. Denote by $K_{p}: \operatorname{Im} L \rightarrow \operatorname{Ker} P \cap \operatorname{dom} L$ the generalised inverse (of $L$ ) and by $J: \operatorname{Im} Q \rightarrow \operatorname{Ker} L$ an isomorphism of $\operatorname{Im} Q$ onto $\operatorname{Ker} L$.

For convenience we introduce a continuation theorem [2, page 40] as follows.

LEMMA 1.1. Let $\Omega \subset X$ be an open bounded set and $N: X \rightarrow Z$ be a continuous operator which is L-compact on $\bar{\Omega}$ (that is, $Q N: \bar{\Omega} \rightarrow Z$ and $K_{p}(I-Q) N: \bar{\Omega} \rightarrow Y$ are compact). Assume

(a) for each $\lambda \in(0,1), x \in \partial \Omega \cap \operatorname{dom} L, L x \neq \lambda N x$;

(b) for each $x \in \partial \Omega \cap \operatorname{Ker} L, Q N x \neq 0$;

(c) $\operatorname{deg}\{J Q N x, \Omega \cap \operatorname{Ker} L, 0\} \neq 0$.

Then $L x=N x$ has at least one solution in $\bar{\Omega}$. 


\section{Main result}

For the sake of convenience we will use the notation

$$
\bar{f}=\frac{1}{w} \int_{0}^{w} f(t) d t, \quad f^{l}=\min _{t \in[0, w]} f(t) \quad \text { and } \quad f^{M}=\max _{l \in[0, w]} f(t),
$$

where $f$ is a strictly positive continuous $w$-periodic function.

We now state our fundamental theorem about the existence of a positive $w$-periodic solution of system (1.2).

THEOREM 2.1. Assume the following:

(i) $\left(a_{1}-D_{1}\right)^{l}>a_{13}^{M} / m^{l}$,

(ii) $a_{31}^{l}>\overline{a_{3}}$,

(iii) $\left(a_{2}-D_{2}\right)^{l}>0$.

Then system (1.2) has at least one positive w-periodic solution.

PROOF. Let

$$
F_{1}(t, s)=\frac{a_{13}(t) e^{y_{3}(s)}}{m(t) e^{y_{3}(s)}+e^{y_{1}(s)}} \quad \text { and } \quad F_{2}(t, s)=\frac{a_{31}(t) e^{y_{1}(s-\tau)}}{m(t) e^{y_{3}(s-\tau)}+e^{y_{1}(s-\tau)}} .
$$

Consider the system

$$
\begin{aligned}
& y_{1}^{\prime}(t)=a_{1}(t)-D_{1}(t)-a_{11}(t) e^{y_{1}(t)}-F_{1}(t, t)+D_{1}(t) e^{y_{2}(t)-y_{1}(t)} \\
& y_{2}^{\prime}(t)=a_{2}(t)-D_{2}(t)-a_{22}(t) e^{y_{2}(t)}+D_{2}(t) e^{y_{1}(t)-y_{2}(t)} \\
& y_{3}^{\prime}(t)=-a_{3}(t)+F_{2}(t, t)
\end{aligned}
$$

where $\tau, D_{i}(i=1,2), a_{i}(i=1,2,3), a_{11}, a_{13}, a_{22}, a_{31}$ and $m$ are the same as those in system (1.2). It is easy to see that if the system (2.1) has an $w$-periodic solution $\left(y_{1}^{*}(t), y_{2}^{*}(t), y_{3}^{*}(t)\right)^{T}$, then $\left(e^{y_{i}^{*}(t)} e^{y_{2}^{*}(t)} e^{y_{j}^{*}(t)}\right)^{T}$ is a positive $w$-periodic solution of system (1.2). Therefore for (1.2) to have at least one positive $w$-periodic solution it is sufficient that (2.1) has at least one $w$-periodic solution. In order to apply Lemma 1.1 to system (2.1), we take

$$
\begin{aligned}
& X=\left\{\left(y_{1}(t), y_{2}(t), y_{3}(t)\right)^{T} \in C^{1}\left(R, R^{3}\right): y_{i}(t+w)=y_{i}(t), \text { for } i=1,2,3\right\} \\
& Z=\left\{\left(z_{1}(t), z_{2}(t), z_{3}(t)\right)^{T} \in C\left(R, R^{3}\right): z_{i}(t+w)=z_{i}(t), \text { for } i=1,2,3\right\}
\end{aligned}
$$

and

$$
\left\|\left(y_{1}(t), y_{2}(t), y_{3}(t)\right)^{T}\right\|=\max _{t \in[0, w]}\left|y_{1}(t)\right|+\max _{t \in[0, w]}\left|y_{2}(t)\right|+\max _{t \in[0, w]}\left|y_{3}(t)\right|
$$


With this norm, $X$ and $Z$ are Banach spaces. Let

$$
\begin{aligned}
N\left[\begin{array}{l}
y_{1} \\
y_{2} \\
y_{3}
\end{array}\right]= & {\left[\begin{array}{c}
a_{1}(t)-D_{1}(t)-a_{11}(t) e^{y_{1}(t)}-F_{1}(t, t)+D_{1}(t) e^{y_{2}(t)-y_{1}(t)} \\
a_{2}(t)-D_{2}(t)-a_{22}(t) e^{y_{2}(t)}+D_{2}(t) e^{y_{1}(t)-y_{2}(t)} \\
-a_{3}(t)+F_{2}(t, t)
\end{array}\right], } \\
L\left[\begin{array}{l}
y_{1} \\
y_{2} \\
y_{3}
\end{array}\right]= & {\left[\begin{array}{l}
y_{1}^{\prime} \\
y_{2}^{\prime} \\
y_{3}^{\prime}
\end{array}\right], \quad P\left[\begin{array}{l}
y_{1} \\
y_{2} \\
y_{3}
\end{array}\right]=\left[\begin{array}{c}
(1 / w) \int_{0}^{w} y_{1}(t) d t \\
(1 / w) \int_{0}^{w} y_{2}(t) d t \\
(1 / w) \int_{0}^{w} y_{3}(t) d t
\end{array}\right], \quad\left[\begin{array}{l}
y_{1} \\
y_{2} \\
y_{3}
\end{array}\right] \in X, } \\
& Q\left[\begin{array}{l}
z_{1} \\
z_{2} \\
z_{3}
\end{array}\right]=\left[\begin{array}{l}
(1 / w) \int_{0}^{w} z_{1}(t) d t \\
(1 / w) \int_{0}^{w} z_{2}(t) d t \\
(1 / w) \int_{0}^{w} z_{3}(t) d t
\end{array}\right], \quad\left[\begin{array}{l}
z_{1} \\
z_{2} \\
z_{3}
\end{array}\right] \in Z .
\end{aligned}
$$

We note that $\operatorname{Ker} L=R^{3}$,

$$
\operatorname{Im} L=\left\{\left[\begin{array}{l}
z_{1} \\
z_{2} \\
z_{3}
\end{array}\right] \mid\left[\begin{array}{l}
z_{1} \\
z_{2} \\
z_{3}
\end{array}\right] \in Z, \int_{0}^{w} z_{i}(t) d t=0, \text { for } i=1,2,3\right\}
$$

is closed in $Z$ and $\operatorname{dim} \operatorname{Ker} L=\operatorname{codim} \operatorname{Im} L=3$. Hence $L$ is a Fredholm mapping of index 0 . Furthermore, the generalised inverse (of $L$ ) $K_{p}: \operatorname{Im} L \rightarrow \operatorname{Ker} P \cap \operatorname{dom} L$ has the form

$$
K_{p}(z)=\int_{0}^{t} z(s) d s-\frac{1}{w} \int_{0}^{w} \int_{0}^{t} z(s) d s d t, \quad \text { for } \quad z=\left[\begin{array}{c}
z_{1} \\
z_{2} \\
z_{3}
\end{array}\right] \in Z .
$$

Thus $Q N: X \rightarrow Z$,

$$
\left[\begin{array}{l}
y_{1} \\
y_{2} \\
y_{3}
\end{array}\right] \rightarrow\left[\begin{array}{c}
\frac{1}{w} \int_{0}^{w}\left[a_{1}(t)-D_{1}(t)-a_{11}(t) e^{y_{1}(t)}-F_{1}(t, t)+D_{1}(t) e^{y_{2}(t)-y_{1}(t)}\right] d t \\
\frac{1}{w} \int_{0}^{w}\left[a_{2}(t)-D_{2}(t)-a_{22}(t) e^{y_{2}(t)}+D_{2}(t) e^{y_{1}(t)-y_{2}(t)}\right] d t \\
\frac{1}{w} \int_{0}^{w}\left[-a_{3}(t)+F_{2}(t, t)\right] d t
\end{array}\right],
$$

$K_{p}(I-Q) N: X \rightarrow X$ and

$$
\begin{aligned}
{\left[\begin{array}{l}
y_{1} \\
y_{2} \\
y_{3}
\end{array}\right] \rightarrow } & {\left[\begin{array}{c}
\int_{0}^{t}\left[a_{1}(s)-D_{1}(s)-a_{11}(s) e^{y_{1}(s)}-F_{1}(s, s)+D_{1}(s) e^{y_{2}(s)-y_{1}(s)}\right] d s \\
\int_{0}^{t}\left[a_{2}(s)-D_{2}(s)-a_{22}(s) e^{y_{2}(s)}+D_{2}(s) e^{y_{1}(s)-y_{2}(s)}\right] d s \\
\int_{0}^{t}\left[-a_{3}(s)+F_{2}(s, s)\right] d s
\end{array}\right] } \\
& -\left[\begin{array}{c}
\frac{1}{w} \int_{0}^{w} \int_{0}^{t}\left[a_{1}(s)-D_{1}(s)-a_{11}(s) e^{y_{1}(s)}-F_{1}(s, s)+D_{1}(s) e^{y_{2}(s) y_{1}(s)}\right] d s d t \\
\frac{1}{w} \int_{0}^{w} \int_{0}^{t}\left[a_{2}(s)-D_{2}(s)-a_{22}(s) e^{y_{2}(s)}+D_{2}(s) e^{y_{1}(s) y_{2}(s)}\right] d s d t \\
\frac{1}{w} \int_{0}^{w} \int_{0}^{t}\left[-a_{3}(s)+F_{2}(s, s)\right] d s d t
\end{array}\right] \\
& -\left(\frac{1}{2}-\frac{t}{w}\right)\left[\begin{array}{c}
\int_{0}^{w}\left[a_{1}(t)-D_{1}(t)-a_{11}(t) e^{y_{1}(t)}-F_{1}(t, t)+D_{1}(t) e^{y_{2}(t)-y_{1}(t)}\right] d t \\
\int_{0}^{w}\left[a_{2}(t)-D_{2}(t)-a_{22}(t) e^{y_{2}}+D_{2}(t) e^{y_{1}(t)-y_{2}(t)}\right] d t \\
\int_{0}^{w}\left[-a_{3}(t)+F_{2}(t, t)\right] d t
\end{array}\right] .
\end{aligned}
$$


Clearly $Q N$ and $K_{p}(I-Q) N$ are continuous by the Lebesgue theorem and moreover $Q N(\bar{\Omega})$ and $K_{p}(I-Q) N(\bar{\Omega})$ are relatively compact for any open bounded set $\Omega \subset X$. Hence $N$ is $L$-compact on $\bar{\Omega}$ for any open bounded set $\Omega \subset X$.

Corresponding to the operator equation $L x=\lambda N x, \lambda \in(0,1)$, we have

$$
\left.\begin{array}{l}
y_{1}^{\prime}(t)=\lambda\left[a_{1}(t)-D_{1}(t)-a_{11}(t) e^{y_{1}(t)}-F_{1}(t, t)+D_{1}(t) e^{y_{2}(t)-y_{1}(t)}\right], \\
y_{2}^{\prime}(t)=\lambda\left[a_{2}(t)-D_{2}(t)-a_{22}(t) e^{y_{2}(t)}+D_{2}(t) e^{y_{1}(t)-y_{2}(t)}\right], \\
y_{3}^{\prime}(t)=\lambda\left[-a_{3}(t)+F_{2}(t, t)\right] .
\end{array}\right\}
$$

Suppose that $\left(y_{1}(t), y_{2}(t), y_{3}(t)\right)^{T} \in X$ is a solution of system (2.2) for a certain $\lambda \in(0,1)$. By integrating $(2.2)$ over the interval $[0, w]$, we obtain

$$
\begin{array}{r}
\int_{0}^{w}\left[a_{1}(t)-D_{1}(t)-a_{11}(t) e^{y_{1}(t)}-F_{1}(t, t)+D_{1}(t) e^{y_{2}(t)-y_{1}(t)}\right] d t=0 \\
\int_{0}^{w}\left[a_{2}(t)-D_{2}(t)-a_{22}(t) e^{y_{2}(t)}+D_{2}(t) e^{y_{1}(t)-y_{2}(t)}\right] d t=0
\end{array}
$$

and

$$
\int_{0}^{w}\left[-a_{3}(t)+F_{2}(t, t)\right] d t=0
$$

Thus

and

$$
\begin{aligned}
\int_{0}^{w}\left[a_{11}(t) e^{y_{1}(t)}+F_{1}(t, t)\right] d t & =\overline{\left(a_{1}-D_{1}\right)} w+\int_{0}^{w} D_{1}(t) e^{y_{2}(t)-y_{1}(t)} d t, \\
\int_{0}^{w} a_{22}(t) e^{y_{2}(t)} d t & =\overline{\left(a_{2}-D_{2}\right)} w+\int_{0}^{w} D_{2}(t) e^{y_{1}(t)-y_{2}(t)} d t
\end{aligned}
$$

$$
\int_{0}^{w} F_{2}(t, t) d t=\overline{a_{3}} w
$$

From (2.2)-(2.5), it follows that

$$
\begin{aligned}
& \int_{0}^{w}\left|y_{1}^{\prime}(t)\right| d t \leq \lambda \int_{0}^{w}\left|a_{1}(t)-D_{1}(t)-a_{11}(t) e^{y_{1}(t)}-F_{1}(t, t)+D_{1}(t) e^{y_{2}(t)-y_{1}(t)}\right| d t \\
& <\overline{\left(a_{1}-D_{1}\right)} w+\int_{0}^{w}\left[a_{11}(t) e^{y_{1}(t)}+F_{1}(t, t)\right] d t \\
& +\int_{0}^{w} D_{1}(t) e^{y_{2}(t)-y_{1}(t)} d t \\
& =2 \overline{\left(a_{1}-D_{1}\right)} w+\int_{0}^{w} D_{1}(t) e^{y_{2}(t)-y_{1}(t)} d t, \\
& \int_{0}^{w}\left|y_{2}^{\prime}(t)\right| d t \leq \lambda \int_{0}^{w}\left|a_{2}(t)-D_{2}(t)-a_{22}(t) e^{y_{2}(t)}+D_{2}(t) e^{y_{1}(t)-y_{2}(t)}\right| d t
\end{aligned}
$$




$$
\begin{aligned}
& <\overline{\left(a_{2}-D_{2}\right)} w+\int_{0}^{w} a_{22}(t) e^{y_{2}(t)} d t+\int_{0}^{w} D_{2}(t) e^{y_{1}(t)-y_{2}(t)} d t \\
& =2 \overline{\left(a_{2}-D_{2}\right)} w+2 \int_{0}^{w} D_{2}(t) e^{y_{1}(t)-y_{2}(t)} d t
\end{aligned}
$$

and

$$
\int_{0}^{w}\left|y_{3}^{\prime}(t)\right| d t \leq \lambda \int_{0}^{w}\left|-a_{3}(t)+F_{2}(t, t)\right| d t<\overline{a_{3}} w+\int_{0}^{w} F_{2}(t, t) d t=2 \overline{a_{3}} w .
$$

Multiplying the first equation and the second equation of system (2.2) by $e^{y_{1}(t)}$ and $e^{y_{2}(t)}$, respectively, and integrating both over $[0, w]$, we obtain

$\int_{0}^{w} e^{y_{1}(t)} y_{1}^{\prime}(t) d t=\int_{0}^{w}\left[\left(a_{1}(t)-D_{1}(t)\right) e^{y_{1}(t)}-a_{11}(t) e^{2 y_{1}(t)}-F_{1}(t, t) e^{y_{1}(t)}+D_{1}(t) e^{y_{2}(t)}\right] d t$ and

$$
\int_{0}^{w} e^{y_{2}(t)} y_{2}^{\prime}(t) d t=\int_{0}^{w}\left[\left(a_{2}(t)-D_{2}(t)\right) e^{y_{2}(t)}-a_{22}(t) e^{2 y_{2}(t)}+D_{2}(t) e^{y_{1}(t)}\right] d t .
$$

That is,

$$
\begin{aligned}
\int_{0}^{w} a_{11}(t) e^{2 y_{1}(t)} d t+\int_{0}^{w} F_{1}(t, t) e^{y_{1}(t)} d t \\
=\int_{0}^{w}\left(a_{1}(t)-D_{1}(t)\right) e^{y_{1}(t)} d t+\int_{0}^{w} D_{1}(t) e^{y_{2}(t)} d t
\end{aligned}
$$

and

$$
\int_{0}^{w} a_{22}(t) e^{2 y_{2}(t)} d t=\int_{0}^{w}\left(a_{2}(t)-D_{2}(t)\right) e^{y_{2}(t)} d t+\int_{0}^{w} D_{2}(t) e^{y_{1}(t)} d t .
$$

Equation (2.9) implies that

$$
a_{11}^{l} \int_{0}^{w} e^{2 y_{1}(t)} d t<\left(a_{1}-D_{1}\right)^{M} \int_{0}^{w} e^{y_{1}(t)} d t+D_{1}^{M} \int_{0}^{w} e^{y_{2}(t)} d t
$$

from which, using the inequality $\left(\int_{0}^{w} e^{y_{1}(t)} d t\right)^{2} \leq w \int_{0}^{w} e^{2 y_{1}(t)} d t$, we obtain

$$
\frac{a_{11}^{l}}{w}\left(\int_{0}^{w} e^{y_{1}(t)} d t\right)^{2}<\left(a_{1}-D_{1}\right)^{M} \int_{0}^{w} e^{y_{1}(t)} d t+D_{1}^{M} \int_{0}^{w} e^{y_{2}(t)} d t
$$

Thus

$$
2 \frac{a_{11}^{l}}{w} \int_{0}^{w} e^{y_{1}(t)} d t<\left[\left(a_{1}-D_{1}\right)^{M}+\left[\left(a_{1}-D_{1}\right)^{M}\right]^{2}+4 \frac{a_{11}^{l} D_{1}^{M}}{w} \int_{0}^{w} e^{y_{2}(t)} d t\right]^{1 / 2}
$$

from which, using the inequality

$$
(a+b)^{1 / 2}<a^{1 / 2}+b^{1 / 2}, \text { for } a>0 \text { and } b>0,
$$


it follows that

$$
\frac{a_{11}^{l}}{w} \int_{0}^{w} e^{y_{1}(t)} d t<\left(a_{1}-D_{1}\right)^{M}+\sqrt{\frac{a_{11}^{l} D_{1}^{M}}{w}}\left(\int_{0}^{w} e^{y_{2}(t)} d t\right)^{1 / 2}
$$

A similar argument to (2.12) implies from (2.10) that

$$
\frac{a_{22}^{l}}{w} \int_{0}^{w} e^{y_{2}(t)} d t<\left(a_{2}-D_{2}\right)^{M}+\sqrt{\frac{a_{22}^{l} D_{2}^{M}}{w}}\left(\int_{0}^{w} e^{y_{1}(t)} d t\right)^{1 / 2}
$$

Substituting (2.13) into (2.12), we obtain

$$
\begin{aligned}
\frac{a_{11}^{l}}{w} \int_{0}^{w} e^{y_{1}(t)} d t< & \left(a_{1}-D_{1}\right)^{M} \\
& +\sqrt{\frac{a_{11}^{l} D_{1}^{M}}{w}}\left[\frac{\left(a_{2}-D_{2}\right)^{M} w}{a_{22}^{l}}+\sqrt{\frac{a_{22}^{l} D_{2}^{M}}{w}} \frac{w}{a_{22}^{l}}\left(\int_{0}^{w} e^{y_{1}(t)} d t\right)^{1 / 2}\right]^{1 / 2},
\end{aligned}
$$

from which, using (2.11), it follows that

$$
\begin{aligned}
\frac{a_{11}^{l}}{w} \int_{0}^{w} e^{y_{1}(t)} d t< & \left(a_{1}-D_{1}\right)^{M} \\
& +\sqrt{\frac{a_{11}^{l} D_{1}^{M}}{a_{22}^{l}}}\left[\left[\left(a_{2}-D_{2}\right)^{M}\right]^{1 / 2}+\sqrt[4]{\frac{a_{22}^{l} D_{2}^{M}}{w}}\left(\int_{0}^{w} e^{y_{1}(t)} d t\right)^{1 / 4}\right]
\end{aligned}
$$

Therefore there exists a positive constant $\rho_{1}$ such that

$$
\int_{0}^{w} e^{y_{1}(t)} d t<\rho_{1}
$$

Substituting (2.14) into (2.13) implies that there exists a positive constant $\rho_{2}$ such that

$$
\int_{0}^{w} e^{y_{2}(t)} d t<\rho_{2}
$$

Choose $t_{i} \in[0, w], i=1,2$, such that $y_{i}\left(t_{i}\right)=\min _{t \in[0, w]} y_{i}(t), i=1,2$. Then it is clear that $y_{i}^{\prime}\left(t_{i}\right)=0, i=1,2$. In view of this and system (2.2), we obtain

$$
a_{1}\left(t_{1}\right)-D_{1}\left(t_{1}\right)-a_{11}\left(t_{1}\right) e^{y_{1}\left(t_{1}\right)}-F_{1}\left(t_{1}, t_{1}\right)+D_{1}\left(t_{1}\right) e^{y_{2}\left(t_{1}\right)-y_{1}\left(t_{1}\right)}=0
$$

and

$$
a_{2}\left(t_{2}\right)-D_{2}\left(t_{2}\right)-a_{22}\left(t_{2}\right) e^{y_{2}\left(t_{2}\right)}+D_{2}\left(t_{2}\right) e^{y_{1}\left(t_{2}\right)-y_{2}\left(t_{2}\right)}=0 \text {. }
$$


Thus

$$
\begin{aligned}
a_{11}^{M} e^{y_{1}\left(t_{1}\right)} & >a_{11}\left(t_{1}\right) e^{y_{1}\left(t_{1}\right)}=a_{1}\left(t_{1}\right)-D_{1}\left(t_{1}\right)-F_{1}\left(t_{1}, t_{1}\right)+D_{1}\left(t_{1}\right) e^{y_{2}\left(t_{1}\right)-y_{1}\left(t_{1}\right)} \\
& >\left(a_{1}-D_{1}\right)^{l}-a_{13}^{M} / m^{l}
\end{aligned}
$$

and

$$
a_{22}^{M} e^{y_{2}\left(t_{2}\right)}>a_{22}\left(t_{2}\right) e^{y_{2}\left(t_{2}\right)}=a_{2}\left(t_{2}\right)-D_{2}\left(t_{2}\right)+D_{2}\left(t_{2}\right) e^{y_{1}\left(t_{2}\right)-y_{2}\left(t_{2}\right)}>\left(a_{2}-D_{2}\right)^{l} .
$$

Therefore

$$
y_{1}\left(t_{1}\right)>\ln \frac{\left(a_{1}-D_{1}\right)^{l}-a_{13}^{M} / m^{l}}{a_{11}^{M}}, \quad y_{2}\left(t_{2}\right)>\ln \frac{\left(a_{2}-D_{2}\right)^{l}}{a_{22}^{M}} .
$$

Substituting (2.14), (2.15) and (2.19) into (2.6) and (2.7), we obtain

$$
\int_{0}^{w}\left|y_{1}^{\prime}(t)\right| d t<2 \overline{\left(a_{1}-D_{1}\right)} w+\frac{2 D_{1}^{M} \rho_{2} a_{11}^{M}}{\left(a_{1}-D_{1}\right)^{l}-a_{13}^{M} / m^{l}} \triangleq d_{1}
$$

and

$$
\int_{0}^{w}\left|y_{2}^{\prime}(t)\right| d t<2 \overline{\left(a_{2}-D_{2}\right)} w+\frac{2 D_{2}^{M} \rho_{1} a_{22}^{M}}{\left(a_{2}-D_{2}\right)^{\prime}} \triangleq d_{2} .
$$

Equations (2.14) and (2.15) imply that there exist two points $\xi, \eta \in(0, w)$ such that

$$
y_{1}(\xi)<\ln \left(\rho_{1} / w\right), \quad y_{2}(\eta)<\ln \left(\rho_{2} / w\right)
$$

In view of this and (2.19), we have

$$
\left|y_{1}(\xi)\right|<\max \left\{\left|\ln \frac{\rho_{1}}{w}\right|,\left|\ln \frac{\left(a_{1}-D_{1}\right)^{l}-a_{13}^{M} / m^{l}}{a_{11}^{M}}\right|\right\}
$$

and

$$
\left|y_{2}(\eta)\right|<\max \left\{\left|\ln \frac{\rho_{2}}{w}\right|,\left|\ln \frac{\left(a_{2}-D_{2}\right)^{l}}{\cdot a_{22}^{M}}\right|\right\} \text {. }
$$

Since $\forall t \in R$

$$
\left|y_{1}(t)\right| \leq\left|y_{1}(\xi)\right|+\int_{0}^{w}\left|y_{1}^{\prime}(s)\right| d s \quad \text { and } \quad\left|y_{2}(t)\right| \leq\left|y_{2}(\eta)\right|+\int_{0}^{w}\left|y_{2}^{\prime}(s)\right| d s,
$$

from (2.20), (2.21) and (2.23), we obtain

and

$$
\left|y_{1}(t)\right|<\max \left\{\left|\ln \frac{\rho_{1}}{w}\right|,\left|\ln \frac{\left(a_{1}-D_{1}\right)^{l}-a_{13}^{M} / m^{l}}{a_{11}^{M}}\right|\right\}+d_{1} \triangleq R_{1}
$$

$$
\left|y_{2}(t)\right|<\max \left\{\left|\ln \frac{\rho_{2}}{w}\right|,\left|\ln \frac{\left(a_{2}-D_{2}\right)^{l}}{a_{22}^{M}}\right|\right\}+d_{2} \triangleq R_{2} .
$$


Equation (2.5) implies that there exists a point $t_{3}^{*} \in(0, w)$ such that

$$
F_{2}\left(t_{3}^{*}+\tau, t_{3}^{*}+\tau\right)=\overline{a_{3}} .
$$

That is, $\overline{a_{3}} m\left(t_{3}^{*}+\tau\right) e^{y_{3}\left(t_{3}^{*}\right)}=\left(a_{31}\left(t_{3}^{*}+\tau\right)-\overline{a_{3}}\right) e^{y_{1}\left(t_{j}^{*}\right)}$. Hence

$$
\left|y_{3}\left(t_{3}^{*}\right)\right|=\left|\ln \frac{a_{31}\left(t_{3}^{*}+\tau\right)-\overline{a_{3}}}{m\left(t_{3}^{*}+\tau\right) \overline{a_{3}}}\right|+\left|y_{1}\left(t_{3}^{*}\right)\right|<\max _{t \in[0, w]}\left|\ln \frac{a_{31}(t)-\overline{a_{3}}}{m(t) \bar{a}_{3}}\right|+R_{1} .
$$

Since $\forall t \in R,\left|y_{3}(t)\right| \leq\left|y_{3}\left(t_{3}^{*}\right)\right|+\int_{0}^{w}\left|y_{3}^{\prime}(s)\right| d s$, from this and (2.8), we obtain

$$
\left|y_{3}(t)\right|<\max _{t \in[0, w]}\left|\ln \frac{a_{31}(t)-\overline{a_{3}}}{m(t) \bar{a}_{3}}\right|+R_{1}+2 a_{3} w \triangleq R_{3} .
$$

Clearly $R_{i}(i=1,2,3)$ are independent of $\lambda$. Denote $M=R_{1}+R_{2}+R_{3}+R_{0}$; here $R_{0}$ is taken sufficiently large such that

$$
\begin{aligned}
& 2 \max \left\{\left|\ln \delta_{1}\right|,\left|\ln \frac{\overline{\left(a_{1}-D_{1}\right)}-\overline{\left(a_{13} / m\right)}}{\overline{a_{11}}}\right|\right\}+\left|\ln \frac{a_{31}^{M}-\overline{a_{3}}}{m^{\prime} \overline{a_{3}}}\right| \\
& \quad+\max \left\{\left|\ln \frac{\overline{\left(a_{2}-D_{2}\right)}+\sqrt{\overline{a_{22}} \overline{D_{2}} \delta_{1}}}{\overline{a_{22}}}\right|,\left|\ln \frac{\overline{\left(a_{2}-D_{2}\right)}}{\overline{a_{22}}}\right|\right\}<M .
\end{aligned}
$$

Here $\sqrt[4]{\delta_{1}}$ is the only real root of the equation

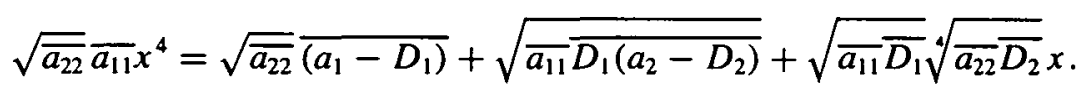

We now take $\Omega=\left\{\left(y_{1}(t), y_{2}(t), y_{3}(t)\right)^{T} \in X:\left\|\left(y_{1}, y_{2}, y_{3}\right)^{T}\right\|<M\right\}$. This satisfies condition (a) of Lemma 1.1. When $\left(y_{1}, y_{2}, y_{3}\right)^{r} \in \partial \Omega \cap \operatorname{Ker} L=\partial \Omega \cap R^{3},\left(y_{1}, y_{2}, y_{3}\right)^{T}$ is a constant vector in $R^{3}$ with $\left|y_{1}\right|+\left|y_{2}\right|+\left|y_{3}\right|=M$. We will prove that when $\left(y_{1}, y_{2}, y_{3}\right)^{T} \in \partial \Omega \cap \operatorname{Ker} L=\partial \Omega \cap R^{3}$,

$$
Q N\left[\begin{array}{l}
y_{1} \\
y_{2} \\
y_{3}
\end{array}\right]=\left[\begin{array}{c}
\overline{\left(a_{1}-D_{1}\right)}-\overline{a_{11}} e^{y_{1}}-\frac{1}{w} \int_{0}^{w} \frac{a_{13}(t)}{m(t) e^{y_{3}}+e^{y_{1}}} d t e^{y_{3}}+\overline{D_{1}} e^{y_{2}-y_{1}} \\
\overline{\left(a_{2}-D_{2}\right)}-\overline{a_{22}} e^{y_{2}}+\overline{D_{2}} e^{y_{1}-y_{2}} \\
-\overline{a_{3}}+\frac{1}{w} \int_{0}^{w} \frac{a_{31}(t)}{m(t) e^{y_{3}}+e^{y_{1}}} d t e^{y_{1}}
\end{array}\right] \neq\left[\begin{array}{l}
0 \\
0 \\
0
\end{array}\right] .
$$

If the conclusion is not true, that is, $Q N\left(y_{1}, y_{2}, y_{3}\right)^{T}=(0,0,0)^{T}$ with $\left|y_{1}\right|+\left|y_{2}\right|+$ $\left|y_{3}\right|=M$. Since

$$
\overline{\left(a_{1}-D_{1}\right)}-\overline{a_{11}} e^{y_{1}}-\frac{1}{w} \int_{0}^{w} \frac{a_{13}(t) d t}{m(t) e^{y_{3}}+e^{y_{1}}} e^{y_{3}}+\overline{D_{1}} e^{y_{2}-y_{1}}=0,
$$

we have $\overline{a_{11}} e^{2 y_{1}}<\overline{\left(a_{1}-D_{1}\right)} e^{y_{1}}+\overline{D_{1}} e^{y_{2}}<\overline{\left(a_{1}-D_{1}\right)} e^{y_{1}}+\overline{D_{1}} e^{y_{2}}$. Thus

$$
2 \overline{a_{11}} e^{y_{1}}<\overline{\left(a_{1}-D_{1}\right)}+\sqrt{\left(\overline{\left.a_{1}-D_{1}\right)}+4 \overline{a_{11}} \overline{D_{1}} e^{y_{2}}\right.}<2 \overline{\left(a_{1}-D_{1}\right)}+2 \sqrt{\overline{a_{11}} \overline{D_{1}}} e^{y_{2} / 2} .
$$


That is,

$$
\overline{a_{11}} e^{y_{1}}<\overline{\left(a_{1}-D_{1}\right)}+\sqrt{\overline{a_{11}} \overline{D_{1}}} e^{y_{2} / 2}
$$

Since

$$
\overline{\left(a_{2}-D_{2}\right)}-\overline{a_{22}} e^{y_{2}}+\overline{D_{2}} e^{y_{1}-y_{2}}=0
$$

we obtain $\overline{a_{22}} e^{2 y_{2}}<\overline{\left(a_{2}-D_{2}\right)} e^{y_{2}}+\overline{D_{2}} e^{y_{1}}$. Thus

$$
\overline{a_{22}} e^{y_{2}}<\overline{\left(a_{2}-D_{2}\right)}+\sqrt{\overline{a_{22}} \overline{D_{2}}} e^{y_{1} / 2} \text {. }
$$

From (2.28) and (2.30), it follows that

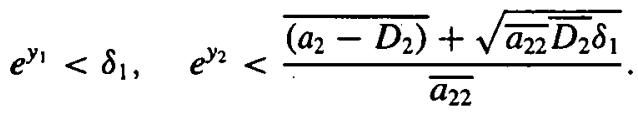

From (2.27) and (2.29), we obtain

$$
e^{y_{1}}>\frac{\overline{\left(a_{1}-D_{1}\right)}-\overline{\left(a_{13} / m\right)}}{\overline{a_{11}}} \text { and } e^{y_{2}}>\frac{\overline{\left(a_{2}-D_{2}\right)}}{\overline{a_{22}}} \text {. }
$$

Hence

$$
\begin{aligned}
& \left|y_{1}\right|<\max \left\{\left|\ln \delta_{1}\right|,\left|\ln \frac{\overline{\left(a_{1}-D_{1}\right)}-\overline{\left(a_{13} / m\right)}}{\overline{a_{11}}}\right|\right\} \text { and } \\
& \left|y_{2}\right|<\max \left\{\left|\ln \frac{\overline{\left(a_{2}-D_{2}\right)}+\sqrt{\overline{a_{22}} \overline{D_{2}} \delta_{1}}}{\overline{a_{22}}}\right|,\left|\ln \frac{\overline{\left(a_{2}-D_{2}\right)}}{\overline{a_{22}}}\right|\right\} .
\end{aligned}
$$

Since $-\overline{a_{3}}+(1 / w) \int_{0}^{w}\left(a_{31}(t) /\left(m(t) e^{y_{3}}+e^{y_{1}}\right)\right) d t e^{y_{1}}=0$, the same argument as that used for (2.25) gives

$$
\left|y_{3}\right| \leq\left|\ln \frac{a_{31}^{M}-\overline{a_{3}}}{m^{l} \overline{a_{3}}}\right|+\max \left\{\left|\ln \delta_{1}\right|,\left|\ln \frac{\overline{\left(a_{1}-D_{1}\right)}-\overline{\left(a_{13} / m\right)}}{\overline{a_{11}}}\right|\right\} .
$$

Therefore

$$
\begin{aligned}
\sum_{i=1}^{3}\left|y_{i}\right| \leq & 2 \max \left\{\left|\ln \delta_{1}\right|,\left|\ln \frac{\overline{\left(a_{1}-D_{1}\right)}-\overline{\left(a_{13} / m\right)}}{\overline{a_{11}}}\right|\right\} \\
& +\max \left\{\left|\ln \frac{\overline{\left(a_{2}-D_{2}\right)}+\sqrt{\overline{a_{22}} \overline{D_{2}} \delta_{1}}}{\overline{a_{22}}}\right|,\left|\ln \frac{\overline{\left(a_{2}-D_{2}\right)}}{\overline{a_{22}}}\right|\right\}+\left|\ln \frac{a_{31}^{M}-\overline{a_{3}}}{m^{l} \overline{a_{3}}}\right| \\
& <M,
\end{aligned}
$$


which contradicts the fact that $\left|y_{1}\right|+\left|y_{2}\right|+\left|y_{3}\right|=M$. So when $\left(y_{1}, y_{2}, y_{3}\right)^{T} \in$ $\partial \Omega \cap \operatorname{Ker} L=\partial \Omega \cap R^{3}, Q N\left(y_{1}, y_{2}, y_{3}\right)^{T} \neq(0,0,0)^{T}$.

Finally we will prove that condition (c) of Lemma 1.1 is satisfied.

Define $\phi: \operatorname{Dom} L \times[0,1] \rightarrow X$ by

$$
\begin{aligned}
\phi\left(y_{1}, y_{2}, y_{3}, \mu\right)= & {\left[\begin{array}{c}
\overline{\left(a_{1}-D_{1}\right)}-\overline{a_{11}} e^{y_{1}} \\
\overline{\left(a_{2}-D_{2}\right)}-\overline{a_{22}} e^{y_{2}} \\
-\overline{a_{3}}+(1 / w) \int_{0}^{w} \frac{a_{31}(t)}{m(t) e^{y_{3}}+e^{y_{1}}} d t e^{y_{1}}
\end{array}\right] } \\
& +\mu\left[\begin{array}{c}
-(1 / w) \int_{0}^{w} \frac{a_{13}(t)}{m(t) e^{e_{3}}+e^{y_{1}}} d t e^{y_{3}}+\overline{D_{1}} e^{y_{2}-y_{1}} \\
\bar{D}_{2} e^{y_{1}-y_{2}} \\
0
\end{array}\right] .
\end{aligned}
$$

When $\left(y_{1}, y_{2}, y_{3}\right)^{T} \in \partial \Omega \cap \operatorname{Ker} L=\partial \Omega \cap R^{3},\left(y_{1}, y_{2}, y_{3}\right)^{T}$ is a constant vector in $R^{3}$ with $\left|y_{1}\right|+\left|y_{2}\right|+\left|y_{3}\right|=M$. Using a similar argument to that for $Q N\left(y_{1}, y_{2}, y_{3}\right)^{T} \neq 0$, when $\left(y_{1}, y_{2}, y_{3}\right)^{T} \in \partial \Omega \cap \operatorname{Ker} L$, we can show that when $\left(y_{1}, y_{2}, y_{3}\right)^{T} \in \partial \Omega \cap \operatorname{Ker} L$, $\phi\left(y_{1}, y_{2}, y_{3}, \mu\right) \neq(0,0,0)^{T}$. As a result, we have

$$
\begin{aligned}
\operatorname{deg}(J & \left.Q N\left(y_{1}, y_{2}, y_{3}\right)^{T}, \Omega \cap \operatorname{Ker} L,(0,0,0)^{T}\right) \\
= & \operatorname{deg}\left(\left(\overline{\left(a_{1}-D_{1}\right)}-\overline{a_{11}} e^{y_{1}}, \overline{\left(a_{2}-D_{2}\right)}-\overline{a_{22}} e^{y_{2}},\right.\right. \\
& \left.\left.-\overline{a_{3}}+\frac{1}{w} \int_{0}^{w} \frac{a_{31}(t)}{m(t) e^{y_{3}}+e^{y_{1}}} d t e^{y_{1}}\right)^{T}, \Omega \cap \operatorname{Ker} L,(0,0,0)^{T}\right) \\
= & \operatorname{deg}\left(\left(\overline{\left(a_{1}-D_{1}\right)}-\overline{a_{11}} e^{y_{1}}, \overline{\left(a_{2}-D_{2}\right)}-\overline{a_{22}} e^{y_{2}},\right.\right. \\
& \left.\left.-\overline{a_{3}}+\frac{\overline{a_{31}} e^{y_{1}}}{m\left(t^{*}\right) e^{y_{3}}+e^{y_{1}}}\right)^{T}, \Omega \cap \operatorname{Ker} L,(0,0,0)^{T}\right),
\end{aligned}
$$

where $t^{*} \in[0, w]$ is a constant.

Since the system of algebraic equations

$$
\left\{\begin{array}{l}
\overline{\left(a_{1}-D_{1}\right)}-\overline{a_{11}} x=0, \\
\overline{\left(a_{2}-D_{2}\right)}-\overline{a_{22}} y=0, \\
-\overline{a_{3}}+\overline{a_{31}} x /\left(m\left(t^{*}\right) z+x\right)=0,
\end{array}\right.
$$

has a unique solution $\left(x^{*}, y^{*}, z^{*}\right)$ which satisfies $x^{*}>0, y^{*}>0$ and $z^{*}>0$, thus

$$
\begin{aligned}
\operatorname{deg}( & \left(\overline{\left(a_{1}-D_{1}\right)}-\overline{a_{11}} e^{y_{1}}, \overline{\left(a_{2}-D_{2}\right)}-\overline{a_{22}} e^{y_{2}},\right. \\
& \left.\left.-\overline{a_{3}}+\frac{\overline{a_{31}} e^{y_{1}}}{m\left(t^{*}\right) e^{y_{3}}+e^{y_{1}}}\right)^{T}, \Omega \cap \operatorname{Ker} L,(0,0,0)^{T}\right)
\end{aligned}
$$




$$
\begin{aligned}
& =\operatorname{sign}\left|\begin{array}{ccc}
-\overline{a_{11}} x^{*} & 0 & 0 \\
0 & -\overline{a_{22}} y^{*} & 0 \\
\overline{a_{31}} m\left(t^{*}\right) z^{*} & 0 & \frac{-m\left(t^{*}\right) \overline{a_{31}} x^{*}}{\left(m\left(t^{*}\right) z^{*}+x^{*}\right)^{2}}
\end{array}\right| \\
& =\operatorname{sign}\left[\frac{-\overline{a_{11}} \overline{a_{22}} m\left(t^{*}\right) \overline{a_{31}} y^{*}\left(x^{*}\right)^{2}}{\left(m\left(t^{*}\right) z^{*}+x^{*}\right)^{2}}\right] \neq 0 .
\end{aligned}
$$

Consequently $\operatorname{deg}\left(J Q N\left(y_{1}, y_{2}, y_{3}\right)^{T}, \Omega \cap \operatorname{Ker} L,(0,0,0)^{T}\right) \neq 0$. This completes the proof of condition (c) of Lemma 1.1.

By now we know that $\Omega$ verifies all the requirements of Lemma 1.1 and that system (2.1) has at least one $w$-periodic solution. Therefore system $(2.1)$ has at least one positive $w$-periodic solution. This completes the proof of Theorem 2.1 .

\section{Acknowledgements}

This project was supported by the NNSF of China (Grant No. 19971026; 10271044).

\section{References}

[1] H. I. Freedman and J. Wu, "Periodic solutions of single-species models with periodic delay", SIAM J. Math. Anal. 23 (1992) 689-701.

[2] R. E. Gaines and J. L. Mawhin, Coincidence degree and non-linear differential equations (Springer, Berlin, 1977).

[3] Y. Kuang, Delay differential equations with applications in population dynamics (Academic Press, New York, 1993).

[4] R. Xu and L. S. Chen, "Persistence and stability for two-species ration-dependent predator-prey system with time delay in a two-patch environment", Comput. Math. Applic. 40 (2000) 577-588. 